\begin{tabular}{c} 
Brazilian Journal \\
of Chemical \\
Engineering \\
\hline
\end{tabular}

ISSN 0104-6632

Printed in Brazil

www.abeq.org.br/bjche

Vol. 33, No. 01, pp. 169 - 175, January - March, 2016

dx.doi.org/10.1590/0104-6632.20160331s20140101

\title{
NUMERICAL MODELING OF HEAVY HYDROCARBON LIQUID HEATING
}

\author{
L. Kovaleva ${ }^{1,2}$ and A. Musin ${ }^{1,2^{*}}$ \\ ${ }^{1}$ Department of Applied Physics, Bashkir State University, Ufa, 450076, Russia. \\ ${ }^{2}$ Center for Micro and Nanoscale Dynamics of Dispersed Systems, Bashkir State University, Ufa, 450076, Russia. \\ E-mail: mus-airat@yandex.ru
}

(Submitted: October 10, 2014 ; Revised: February 12, 2015 ; Accepted: March 6, 2015)

\begin{abstract}
The results of two-dimensional mathematical modeling of heat and mass transfer in a highly viscous hydrocarbon liquid by inductive and radio-frequency (RF) electromagnetic (EM) heating are presented. The model takes into account the dependence of the liquid's viscosity and thermal conductivity on the temperature and also the presence of thermal convection effects. It is shown that the occurrence of volumetric heat sources inside the liquid caused by EM radiation yields an intensive deep heating as compared with inductive heating. Numerical calculations show that, in both these cases, the single vortex flow structure is formed in the whole volume of the liquid. However, RF EM heating provides a more homogeneous distribution of heat in the medium and requires three-fold less power consumption in comparison with induction heating.

Keywords: Radio-frequency electromagnetic field; Induction heating; Hydrocarbon liquid; Thermal convection; Heat transfer; Mathematical model.
\end{abstract}

\section{INTRODUCTION}

The study of convective heat transfer in multiphase multicomponent media, such as natural (oil and bitumen) or manmade (sludge and petroleum products) hydrocarbon systems, are associated with a number of problems arising in the oil and gas industry. One of them is the utilization of oil sludge, formed as a result of tank and reservoir cleaning. Heating significantly reduces the viscosity of such systems and may cause convective motion of the liquid (Kovaleva et al., 2005, 2010). The area covered by the motion, as well as the temperature field in this area can continuously and irregularly change. There are various ways of transfering heat energy from the source to the medium and inductive and electromagnetic heating are among these. In recent years, the use of high-frequency changing EM fields has become a widely used technology for heating complex hydrocarbon systems (Carrizales et al., 2008; Kasevich et al., 1994; Kovaleva et al., 2008, 2009, 2011a, 2011b; Bientinesi et al., 2013). The urgency of this technology is connected with the fact that, for the considered media, it is important not only to reduce the viscosity by heating, but to a greater extent to destroy stable structures of high-molecular hydrocarbon compounds by the effect of electromagnetic radiation. The most effective method of applying heat and also provoking a "destructive" effect in such media is by heating with a radio-frequency electromagnetic field. The advantage of this method of heating as compared with the other types of treatment is that it involves a large volume of liquid and the speed of the process is very high.

The results of a numerical study of the processes of heat and mass transfer in a hydrocarbon liquid under the influence of the induction and RF EM heaters are given in the paper. The results are obtained by the solution of the full system of equations of thermal convection, taking into account the dependence

*To whom correspondence should be addressed 
of the thermo-physical parameters on the temperature of the liquid.

\section{PROBLEM STATEMENT}

We have studied the thermal motion of a heavy hydrocarbon liquid with temperature-dependent viscosity and thermal conductivity in the space between two coaxial cylindrical tubes using various methods of heating. The reservoir model consists of two coaxial metal tubes ( 1 and 2 in Figure 1), the lower part of which is closed by a plug (4). The space between the tubes is filled with the hydrocarbon liquid (3).

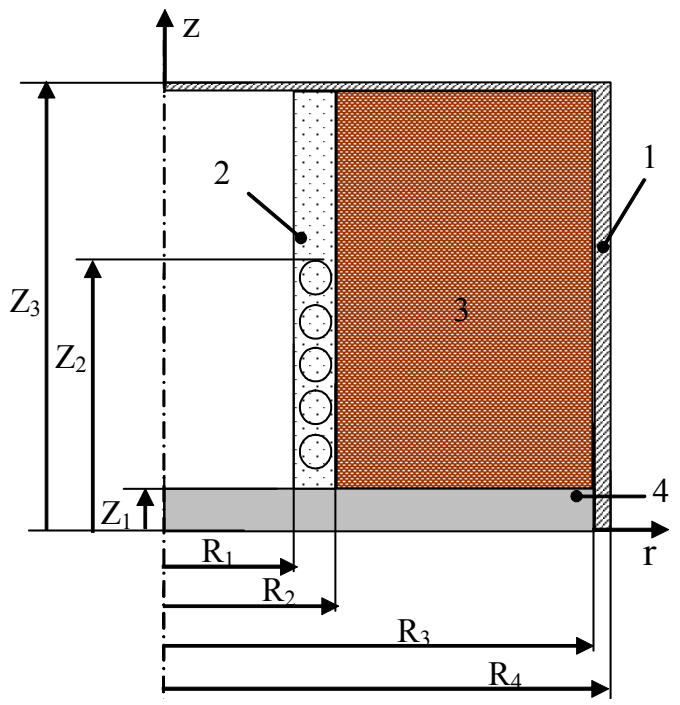

Figure 1: Scheme of the experimental zone: 1) reservoir wall; 2) inner tube; 3) hydrocarbon liquid; 4) bottom of the reservoir.

The selected geometry of the physical model, from the electrodynamics point of view, is a cylindrical capacitor, which gives the possibility to use different methods of heating of the high-viscosity hydrocarbon medium. Two of these methods differ not only in the way of implementation, but also in the nature of the forces leading to heating of the medium:

1. Induction (or contact) heating, i.e., heating of the liquid filling the annular space of the reservoir with an induction heater placed at its center. In this case heating is carried out by heat applied on the inner tube.

2. Heating of the liquid by exposure to radio-frequency electromagnetic field (RF EMF) is due to EMF energy dissipation in the liquid (volumetric heating). The outer tube of the reservoir and the inner tube in this case act as electrodes.

\section{MATHEMATICAL MODEL}

A mathematical model of the impact on the hydrocarbon medium includes the system of equations of mass conservation, energy and momentum. From the point of view of mathematical modeling, the physical model is a multi-layer system: a reservoir wall (outer tube), an inner tube, a hydrocarbon liquid, concrete bottom of the reservoir.

The distribution of temperature in the solid parts of the model (1, 2 and 4 in Figure 1) is described by the thermal conductivity equation:

$\rho_{i} c_{i} \frac{\partial T}{\partial t}=k_{i} \nabla^{2} T+q_{i}$

where $\rho_{i}, c_{i}, k_{i}$ are density, heat capacity and coefficient of thermal conductivity of the $i$-th material, respectively; $q_{i}$ is the density of the distributed heat sources in the $i$-th material; $I=1,2,4$.

Temperature and velocity fields in the layer of liquid are described by the system of equations of thermal convection in the Boussinesq approximation (Gershuni and Zhukhovitsky, 1976):

$$
\begin{aligned}
& \rho_{3}\left(\frac{\partial \mathbf{v}}{\partial t}+(\mathbf{v} \nabla) \mathbf{v}\right)=-\nabla P+\nabla\left(\eta_{3} \nabla \mathbf{v}\right)+\rho_{3} \mathbf{f} \\
& \rho_{3} c_{3}\left(\frac{\partial T}{\partial t}+(\mathbf{v} \nabla) T\right)=\nabla\left(k_{3} \nabla T\right)+q_{3} \\
& \nabla \mathbf{v}=0
\end{aligned}
$$

Here $\rho_{3}, \eta_{3}, c_{3}, k_{3}$ are density, coefficient of dynamic viscosity, heat capacity and thermal conductivity of a hydrocarbon liquid; $\mathbf{v}$ is a vector field of velocity of free convective motion of the liquid; $P$ is pressure; $T$ is temperature; $\mathbf{f}$ is the vector field of mass forces; $q_{3}$ is the density of distributed heat sources in the liquid.

In the given mathematical model (2)-(4) the following assumptions have been made:

1) the medium is homogeneous, isotropic and incompressible;

2) electrodynamic and thermal properties except for the viscosity and the thermal conductivity are constant;

3 ) in the equations of motion, the effect of terms containing the derivatives of viscosity with respect to temperature is negligible; 
4) the temperature change caused by heat generation due to the dissipation of energy by internal friction is negligible;

5) the flow is laminar.

\section{Additional Relations}

The thermal motion of the liquid occurs in the gravitational field in the presence of spatial heterogeneity of density caused by the inhomogeneity of the temperature (Gershuni and Zhukhovitsky, 1976). The density, temperature, and pressure of the medium are interconnected through the equation of state $\rho=\rho(T, P)$. The form of this dependence for different media is different and depends on many factors, including the magnitude of the changes of temperature and pressure. Hydrocarbon liquid is a complex heterogeneous system. Its composition and its physico-chemical properties can vary within very wide limits. In particular, it concerns the value of the boiling point of the liquid. Every component of a hydrocarbon medium has its own boiling point and, in the process of heating, their transition into the gaseous state occurs at different temperatures. Therefore, it is natural to assume that, in the process of heating, there can be simultaneously two phases in one hydrocarbon medium: gas and liquid. Moreover, the concentration of the gaseous phase rises with the temperature increase.

In the general case, for the simulation of such a system, the two-phase nature of the medium should be taken into account and the transfer equations should be solved for each of the phases. However, if we assume that the gas and liquid phases behave as a single system and if we neglect the compressibility of the medium, the quasi-homogeneous approximation can be applied (Kutateladze and Nakoryakov, 1984). In this case, it is possible to solve the system of equations mentioned above using effective values of some of the physical quantities: density, coefficient of dynamic viscosity, coefficient of thermal conductivity, heat capacity, and the averaged values of velocity, temperature and pressure in the medium.

The density of the mixture is assumed to be an additive quantity, while the concentration of the gas phase is associated with intensity of degassing of the hydrocarbon system, which depends on the temperature. Then the dependence of the mixture density on temperature can be represented by the following empirical relation:

$$
\rho_{3 s}=\rho_{3}\left(1-B \exp \left(-\frac{\gamma}{T-T_{s}}\right)\right)
$$

Here $T$ is the current temperature, $T_{s}=70{ }^{\circ} \mathrm{C}$ is the temperature of the beginning of oil degassing, $B=$ 0.0542 and $\gamma=93{ }^{\circ} \mathrm{C}$ are the empirical coefficients of the degassing process intensity. Then, following the Boussinesq approximation, the components of the mass forces $\mathbf{f}$ in equation (2) under the conditions of thermal convection will be present in the following form:

$f_{r}=0, \quad f_{z}=g B \exp \left(-\frac{\gamma}{\left(T-T_{s}\right)}\right)$

where $g$ is the acceleration due to gravity.

The viscosity of the hydrocarbon liquid is also temperature dependent: the approximate dependence of viscosity on temperature in the form of two experimentally obtained exponents has been used (Kovaleva et al., 2005):

$\eta_{3}(T)= \begin{cases}\eta_{01} \exp \left(-\gamma_{1}\left(T-T_{01}\right)\right), & T_{01}<T<T_{02}, \\ \eta_{02} \exp \left(-\gamma_{2}\left(T-T_{02}\right)\right), & T_{02}<T<T_{03},\end{cases}$

where $\eta_{01}=1460 \mathrm{~Pa} \cdot \mathrm{sec}$ is the viscosity of the hydrocarbon liquid at the temperature of $T_{01}=35.5{ }^{\circ} \mathrm{C}$; $\eta_{02}=0.228 \mathrm{~Pa} \cdot \mathrm{sec}$ is the viscosity of the hydrocarbon liquid at the temperature of $T_{02}=54.2{ }^{\circ} \mathrm{C} ; \gamma_{1}=$ $0.497 \mathrm{~K}^{-1}$ is the temperature coefficient within the range $T_{01}<T<T_{02} ; \gamma_{2}=0.031 \mathrm{~K}^{-1}$ is the temperature coefficient within the $T>T_{02}$ range.

The dependence of the conductivity coefficient of the liquid on the temperature was set as linear (Kovaleva et al., 2005):

$k_{3}(T)=k_{30}\left[1+b\left(T-T_{0}\right)\right]$.

Here $k_{30}$ is the coefficient of thermal conductivity at the temperature of $T=T_{0} ; b=0.0095 \mathrm{~K}^{-1}$ is an empirical constant.

The definition of the ratios for the density of distributed heat sources $q$ in equations (1), (3) and their values depends on the type of heating:

- induction (contact) heating is modeled by placing the distributed sources of heat on the inner tube of the reservoir (2 in Figure 1) (Kovaleva et al., 2005):

$$
q_{2}=\frac{N_{I}}{\pi\left(R_{2}^{2}-R_{1}^{2}\right)\left(Z_{2}-Z_{1}\right)}, q_{1}=q_{3}=q_{4}=0
$$


where $N_{I}$ is the net power of the inductor; $R_{1}, R_{2}$ are the inner and outer radii of the inductor tubes; $Z_{2}-Z_{1}$ is the height of the inductor.

- RF EM heating is modeled by placing a distributed heat source in the liquid (3 in Figure 1). For a cylindrical capacitor the following equation is used (Landau and Lifshitz, 1960):

$$
q_{3}=\frac{N_{R}}{\pi r^{2}\left(Z_{3}-Z_{2}\right) \ln \frac{R_{3}}{R_{2}}}, \quad q_{1}=q_{2}=q_{4}=0
$$

where $N_{R}$ is the net power of the RF generator; $r$ is radius-vector; $R_{3}$ is the radius of the reservoir; $Z_{3}-Z_{2}$ is the reservoir depth.

\section{Boundary Conditions}

Initially, the liquid is motionless and the model has a constant temperature equal to the ambient:

$$
\begin{aligned}
& u(x, y, 0)=v(x, y, 0)=0 \\
& T(r, z, 0)=T_{0}
\end{aligned}
$$

Adhesion conditions are set for all velocities at solid walls:

$$
\left.u\right|_{G}=\left.v\right|_{G}=0
$$

All the internal borders of the reservoir are marked by the index "G".

In accordance with the terms of the experiments, the following boundary conditions were chosen:

- in the case of induction heating, the condition of the heat exchange with the environment on the upper surface of the reservoir is given according to the law of free convection in an unbounded space. The outer wall of the reservoir and its lower base are heat insulated. The condition of heat exchange with the air in a confined space is applied on the inner surface of the inductor tube. The corresponding boundary conditions are:

$$
\begin{aligned}
& \alpha_{h}\left(T\left(r, Z_{3}, t\right)-T_{0}\right)=-k_{i} \frac{\partial T\left(r, Z_{3}, t\right)}{\partial z}, \\
& \alpha_{v}\left(T\left(R_{1}, z, t\right)-T_{0}\right)=-k_{i} \frac{\partial T\left(R_{1}, z, t\right)}{\partial r} \\
& \frac{\partial T\left(R_{3}, z, t\right)}{\partial r}=0, \frac{\partial T(r, 0, t)}{\partial z}=0 .
\end{aligned}
$$

Here $\alpha_{h}, \alpha_{v}$ are heat transfer coefficients along the horizontal and vertical walls; $T_{0}$ is the initial temperature of the medium and the temperature of the air surrounding the experimental unit.

- In the case of RF EM heating, the reservoir with liquid is heat insulated on all sides. The corresponding boundary conditions are:

$$
\begin{aligned}
& \frac{\partial T(r, 0, t)}{\partial z}=0, \frac{\partial T\left(r, Z_{3}, t\right)}{\partial z}=0 \\
& \frac{\partial T(0, z, t)}{\partial r}=0, \frac{\partial T\left(R_{4}, z, t\right)}{\partial r}=0 .
\end{aligned}
$$

\section{RESULTS OF CALCULATIONS}

The system of Equations (1)-(4) with the closing ratios (5-14) was solved numerically by the method of control volume with the help of the SIMPLE algorithm (Patankar, 1980).

The numerical values of the physical and geometrical parameters of the model were taken in accordance with the parameters of laboratory facilities used in experimental research. Model properties are given in Table 1.

Values of the unknown quantities were determined by comparing the results of mathematical modeling with experimental results.

Table 1: Model properties.

\begin{tabular}{|l|c|c|}
\hline Properties & $\begin{array}{c}\text { Value for Induction } \\
\text { heating }\end{array}$ & $\begin{array}{c}\text { Value for RF EM } \\
\text { heating }\end{array}$ \\
\hline$k_{1}, \mathrm{~W} /(\mathrm{m} \cdot \mathrm{K})$ & 45 & 45 \\
$k_{2}, \mathrm{~W} /(\mathrm{m} \cdot \mathrm{K})$ & 45 & 120 \\
$k_{3}, \mathrm{~W} /(\mathrm{m} \cdot \mathrm{K})$ & 0.125 & 0.125 \\
$k_{4}, \mathrm{~W} /(\mathrm{m} \cdot \mathrm{K})$ & 0.279 & 45 \\
$c_{1}, \mathrm{~J} /(\mathrm{kg} \cdot \mathrm{K})$ & 461 & 461 \\
$c_{2}, \mathrm{~J} /(\mathrm{kg} \cdot \mathrm{K})$ & 461 & 920 \\
$c_{3}, \mathrm{~J} /(\mathrm{kg} \cdot \mathrm{K})$ & 1864 & 1864 \\
$c_{4}, \mathrm{~J} /(\mathrm{kg} \cdot \mathrm{K})$ & 1130 & 461 \\
$\rho_{1}, \mathrm{~kg} / \mathrm{m}^{3}$ & 7900 & 7900 \\
$\rho_{2}, \mathrm{~kg} / \mathrm{m}^{3}$ & 7900 & 2750 \\
$\rho_{3}, \mathrm{~kg} / \mathrm{m}^{3}$ & 954 & 954 \\
$\rho_{4}, \mathrm{~kg} / \mathrm{m}^{3}$ & 2300 & 7900 \\
$Z_{1}, \mathrm{~m}$ & 0.040 & 0.010 \\
$Z_{2}, \mathrm{~m}$ & 0.290 & 0.010 \\
$Z_{3}, \mathrm{~m}$ & 0.390 & 0.440 \\
$R_{1}, \mathrm{~m}$ & 0.074 & 0.000 \\
$R_{2}, \mathrm{~m}$ & 0.080 & 0.010 \\
$R_{3}, \mathrm{~m}$ & 0.225 & 0.104 \\
$R_{4}, \mathrm{~m}$ & 0.225 & 0.110 \\
$N, \mathrm{~W}$ & 1224 & 260 \\
$T_{0},{ }^{\circ} \mathrm{C}$ & 24 & 24 \\
\hline
\end{tabular}

In the process of mathematical modeling, temperature fields and streamlines in a hydrocarbon 
liquid heated by induction and RF EM sources were obtained. Low temperature and high viscosity of the liquid at the initial time exclude the possibility of any motion of the liquid. Therefore, for a sufficiently long time after the start of the experiment, the medium is heated only by the distributed heat sources and the mechanism of molecular heat conduction. Then the zone warmed up to the temperature of fluidity is formed near the inner tube (rod) and that is sufficient for the emergence of the thermal motion of the liquid and the mechanism of convective heat transfer becomes part of the heating process. It is revealed that in both cases a single vortical flow structure is observed in the whole volume of the liquid (Figure 2). Most of the time the core of the flow is located in the upper part of the reservoir. By the end of the process it is shifted to the center of the reservoir. The asymmetry of the main flow that is observed throughout the heating process is the result of the clearly defined
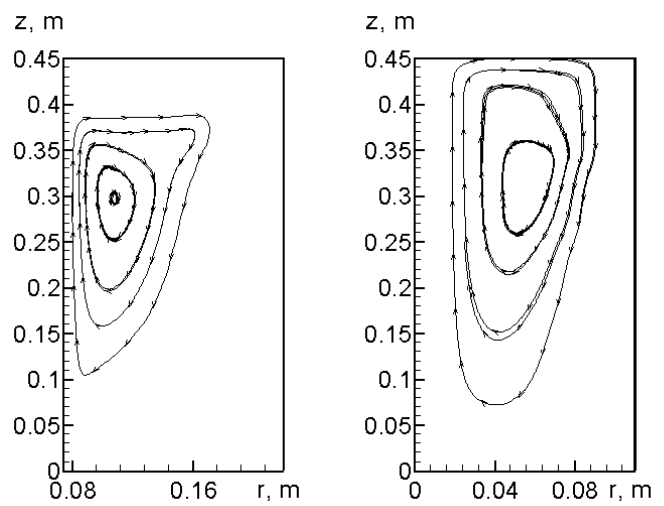

Figure 2: Streamlines in the liquid at the 80th minute of the experiment: a) induction heating, b) RF EM heating.

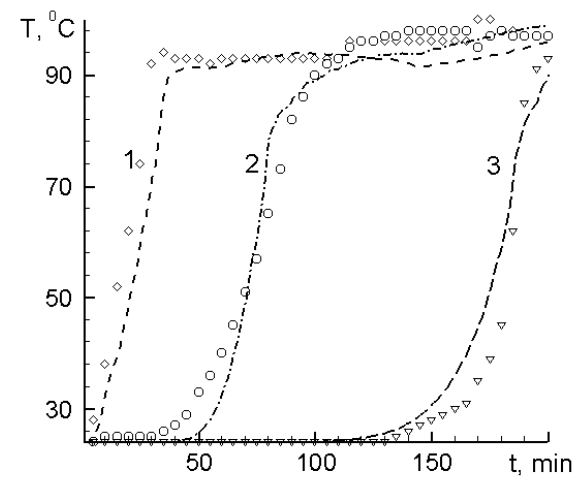

Figure 4: Dynamics of the temperature distribution in the reservoir liquid during induction heating: symbols - experimental data; lines - calculated data; curves 1,2, 3 correspond to the positions of the first, second and third thermocouples. dependence of viscosity on temperature.

The intensity of expansion of the zone heated to the fluidity temperature where convective motion is taking place is relatively higher in the case of RF electromagnetic heating than in the case of induction heating (Figure 3). Due to a more homogeneous nature of the temperature distribution under RF EM heating, a large part of the volume of the liquid is covered with a convective flow as compared to induction heating. This in its turn significantly speeds up the process of heat transfer in the liquid.

The main difference between RF electromagnetic and induction heating is that the electromagnetic heating covers a large volume of liquid. The distinctive feature of RF EM heating can be seen through the comparison of the dynamics of the temperature change measured with thermocouples located at different distances from the axis of the reservoir during induction (Figure 4) and RF EM heating (Figure 5).
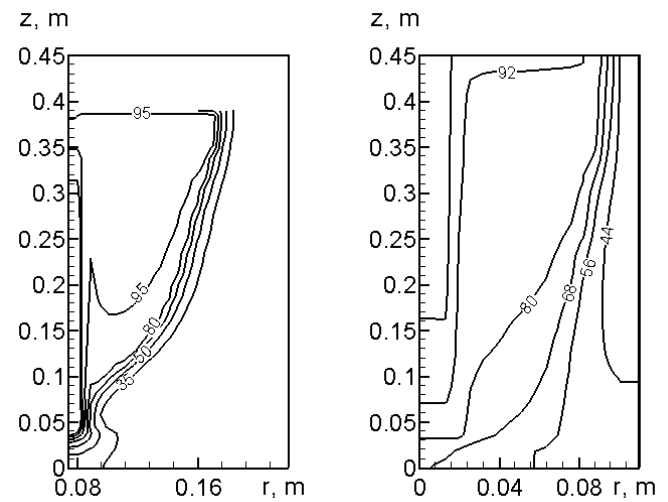

Figure 3: Temperature zones in the liquid at the 80th minute of the experiment: a) induction heating, b) RF EM heating.

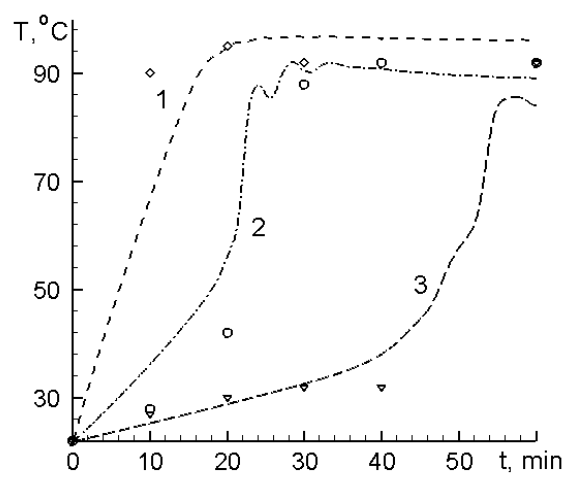

Figure 5: Dynamics of changes in temperature of the liquid in the reservoir in the process of RF EM heating: symbols - experimental data; lines calculated data; curves 1, 2, 3 represent readings on $1,2,3$ thermocouples respectively. 
It is shown in Figure 4 that, in the process of induction heating, the temperature increases first on the surface of the inner tube (curve 1 in Fig. 4) and only after 50 minutes is a consistent rise in temperature observed on the second (curve 2) and the third (curve 3) thermocouples. In the case of RF electromagnetic heating (Fig. 5), the temperature begins to rise with varying intensity simultaneously on all thermocouples (curves 1, 2 and 3 in Fig. 5). This occurs due to the fact that the more homogeneous redistribution of the energy in the case of RF EM heating prevents the local overheating of the liquid that takes place in induction heating and a steady temperature rise is observed throughout the volume of the liquid. After reaching the temperature of fluidity, a larger part of the liquid is covered by a convection flow, thereby increasing its heating volume. Points in Figs. 4 and 5 show the temperature readings on the thermocouples obtained in the process of laboratory experimental studies.

\section{CONCLUSION}

The comparative efficiency of radio-frequency electromagnetic field heating in comparison with induction heating of a heavy hydrocarbon liquid system was shown by mathematical modeling. It was proved that, in both cases, a single vortex flow structure is formed in the whole volume of the liquid, which expands as the liquid reaches the fluidity temperature. However, RF EM heating provides a more homogeneous redistribution of heat and prevents local overheating of the liquid, which is observed in the process of induction heating. Besides, in the case of induction heating, a three-fold lower power consumption is required for adequate heating; this fact indicates that RF electromagnetic heating is significantly more efficient when dealing with high-viscosity hydrocarbon liquids in comparison with induction heating.

\section{ACKNOWLEDGMENT}

This research is supported by the Ministry of Education and Science of the Russian Federation (government contract No.3.1251.2014/K), the RFBR grant № 14-01-97005 and the Grant of President of the Russian Federation № MK-9398.2016.1.

\section{NOMENCLATURE}

$B \quad$ the empirical coefficient of the degassing process intensity

$b \quad$ empirical constant

\begin{tabular}{|c|c|}
\hline$c_{i}$ & heat capacity of the $i$-th material \\
\hline f & vector field of mass forces \\
\hline & acceleration due to gravity \\
\hline$k_{i}$ & $\begin{array}{l}\text { coefficient of thermal conductivity of the } \\
i \text {-th material }\end{array}$ \\
\hline$k_{30}$ & $\begin{array}{l}\text { coefficient of thermal conductivity at the } \\
\text { temperature of } T=T_{0}\end{array}$ \\
\hline$N_{I}$ & net power of inductor \\
\hline$N_{R}$ & net power of RF generator \\
\hline$P$ & pressure \\
\hline$q_{i}$ & $\begin{array}{l}\text { the density of the distributed heat sources } \\
\text { in the } i \text {-th material }\end{array}$ \\
\hline$R_{1}$ & inner radius of the inductor tubes \\
\hline$R_{2}$ & outer radius of the inductor tubes \\
\hline$R_{3}$ & radius of the reservoir \\
\hline & radius-vector \\
\hline$T$ & temperature \\
\hline$T_{0}$ & $\begin{array}{l}\text { initial temperature of the medium and the } \\
\text { temperature of the air surrounding the } \\
\text { experimental unit }\end{array}$ \\
\hline$T_{s}$ & $\begin{array}{l}\text { temperature of the beginning of oil } \\
\text { degassing }\end{array}$ \\
\hline $\mathbf{v}$ & $\begin{array}{l}\text { a vector field of velocity of free convective } \\
\text { motion of liquid } \mathbf{v}=(u, v)\end{array}$ \\
\hline$Z_{1}$ & height of the bottom of the reservoir \\
\hline$Z_{2}$ & height of the inductor \\
\hline$Z_{3}$ & height of the reservoir \\
\hline
\end{tabular}

\section{Greek Symbols}

$\alpha_{h} \quad$ heat transfer coefficient along the horizontal walls

$\alpha_{v} \quad$ heat transfer coefficient along the vertical walls

$\gamma \quad$ the empirical coefficient of the degassing process intensity

$\gamma_{1}$ temperature coefficient within the range $T_{01}<T<T_{02}$

$\gamma_{2}$ temperature coefficient within the $T>T_{02}$ range

$\eta_{01} \quad$ viscosity of the hydrocarbon liquid at the temperature of $T_{01}$

$\eta_{02}$ viscosity of the hydrocarbon liquid at the temperature of $T_{02}$

$\eta_{3} \quad$ coefficient of dynamic viscosity of the hydrocarbon liquid

$\rho_{i} \quad$ density of the $i$-th material

\section{Subscripts}

$i \quad$ the index of material No $1,2,3$ and 4

$\mathrm{G}$ all the internal borders of the reservoir 


\section{REFERENCES}

Bientinesi, M., Petarca, L., Cerutti, A., Bandinelli, M., De Simoni, M., Manotti, M., Maddinelli, G., A radiofrequency microwave heating method for thermal heavy oil recovery based on a novel tight-shell conceptual design. Journal of Petroleum Science and Engineering, v. 107, pp. 18-30 (2013).

Carrizales, M. A., Lake, L. W. and Johns, R. T., Production improvement of heavy-oil recovery by using electromagnetic heating. Colorado, USA, 21-24 September, SPE 115723 (2008).

Gershuni, G. Z. and Zhukovitskii, E. M., Convective Stability of Incompressible Fluids. Nauka, Moscow (1972). Translated from the Russian by Louvish D. Keter Publications, Jerusalem/Wiley, p. 330 (1976).

Kasevich, R. S., Price, S. L., Faust, D. L. and Fontaine, M. F., Pilot testing of a radio frequency heating system for enhanced oil recovery from diatomaceous earth. 69 Annual Technicel Conference and Exhibition held in New Orleans, USA, SPE 28619, pp. 105-118 (1994).

Kovaleva, L. A., Kireev, V. N. and Musin, A. A., Modeling of heat and mass transfer in a hydrocarbon fluid under inductive heating. Journal of Applied Mechanics and Technical Physics, 50(1), pp. 80-85 (2009).

Kovaleva, L. A., Minnigalimov, R. Z. and Zinnatullin,
R. R., Destruction of water-in-oil emulsions in radio-frequency and microwave electromagnetic fields. Energy and Fuels, 25(8), 3731-3738 (2011a).

Kovaleva, L. A., Minnigalimov, R. Z. and Zinnatullin, R. R., Development of electromagnetic technology for recycling oil slimes. Oil Industry, 9, pp. 75-79 (2009).

Kovaleva, L., Musin, A., Zinnatullin, R. and Akhatov, I. S., Destruction of water-in-oil emulsions in electromagnetic fields. Proceedings of ASME 2011 International Mechanical Engineering Congress \& Exposition, IMECE2011-62935, Denver, Colorado, USA, November 11-17 (2011b).

Kovaleva, L. A., Nasyrov, N. M., Maksimochkin, V. I. and Sufyanov, R. R., Experimental and numerical modeling of the thermal conductivity of high-viscosity hydrocarbon systems. Journal of Applied Mechanics and Technical Physics, 46(6), pp. 851-856 (2005).

Kutateladze, S. S. and Nakoryakov, V. E., Teplomassoobmen $\mathrm{i}$ volny $\mathrm{V}$ gazozhidkostnykh sistemakh (Heat and mass transfer and waves in gas-liquid systems). Novosibirsk, Nauka Publishers, p. 301 (1984).

Landau, L. D. and Lifshitz, E. M., Electrodynamics of Continuous Media (Volume 8 of A Course of Theoretical Physics), Pergamon Press (1960).

Patankar, S., Numerical Heat Transfer and Fluid Flow. Hemisphere Publishing Corporation, New York (1980). 